\title{
Etude Des Parametres Floraux De Quatre Varietes L’abricotier Dans La Region De Guedila (Biskra, Algerie)
}

\author{
Benaziza Abdelaziz \\ Makhloula Samiha
}

Département des sciences agronomiques, Université de Biskra, Algérie doi: 10.19044/esj.2016.v13n3p461 URL:http://dx.doi.org/10.19044/esj.2016.v13n3p461

\begin{abstract}
In the Guedila region (Biskra, Algeria), fruit trees have become increasingly important in recent years, occupying enormous areas and important plant resources. Among these, apricot tree (Prunus armeniaca L.) or (Armeniaca vulgaris Lam.). Which is undergoing a notable development due to the good adaptation of this species to the regional pedoclimatic conditions which generate very satisfactory yields?

This work allows the floral characterization of four varieties of apricot, most cultivated in this region. It is based mainly on the descriptor of the European union of production and plant breeding (UPOV, 2008) which allows varietal identification, their performances and the existing analogies between the varieties studied.
\end{abstract}

Keywords: phenology, Apricot tree, descriptor, Biskra, Algeria.

\section{Résumé}

L'arboriculture fruitière se démarque ces dernières années dans la région de Guedila (Biskra, Algérie) par un essor considérable, elle occupe d'énormes superficies et des ressources végétales importantes. Parmi cellesci l'abricotier (Prunus armeniaca L.) ou (Armeniaca vulgaris Lam.) qui connaît un développement notable dû à la bonne adaptation de cette espèce aux conditions pédoclimatiques régionales qui engendrent des rendements très satisfaisants.

Le présent travail permet la caractérisation florale de quatre variétés d'abricotier les plus cultivées dans cette région. Il est basé principalement sur le descripteur de l'union européenne de la production et l'obtention végétale (UPOV, 2008) qui permet l'identification variétale, leurs performances et les analogies existantes entre les variétés étudiées. 
Mots clés : phénologie, Abricotier, descripteur, Biskra, Algérie.

\section{Introduction :}

L'arboriculture fruitière notamment les espèces à noyaux connaissent au sud-est algérien durant cette décennie un essor certain. Parmi celle-ci l'abricotier qui se démarque par un développement remarquable et une diversité variétale appréciable (D.SA, 2013).

D'après la F. A .O (2013) cette espèce prend une croissance importante et se distingue par une adaptation particulière aux conditions méditerranéennes où sa culture présente un intérêt socioéconomique local et s'adapte à certains étages bioclimatiques des oasis présahariennes.

Dans l'optique d'une meilleure caractérisation variétale dans ces zones, cette première étude vise la connaissance du patrimoine arboricole existant dans la wilaya de Biskra (sud-est d'Algérie). Elle est axée sur les critères floraux et l'évaluation des rendements de quatre variétés les plus dominantes dans la zone d'étude.

\section{Matériel végétal :}

Le matériel végétal utilisé est composé de quatre variétés d’abricotier les plus cultivées dans la région d'étude (figure 1). Ces variétés sont essentiellement Arbi, Boufarik, Badai et Pêche de Nancy en étudiant leurs critères phénologiques et l'évaluation des rendements dans ces situations pédoclimatiques.

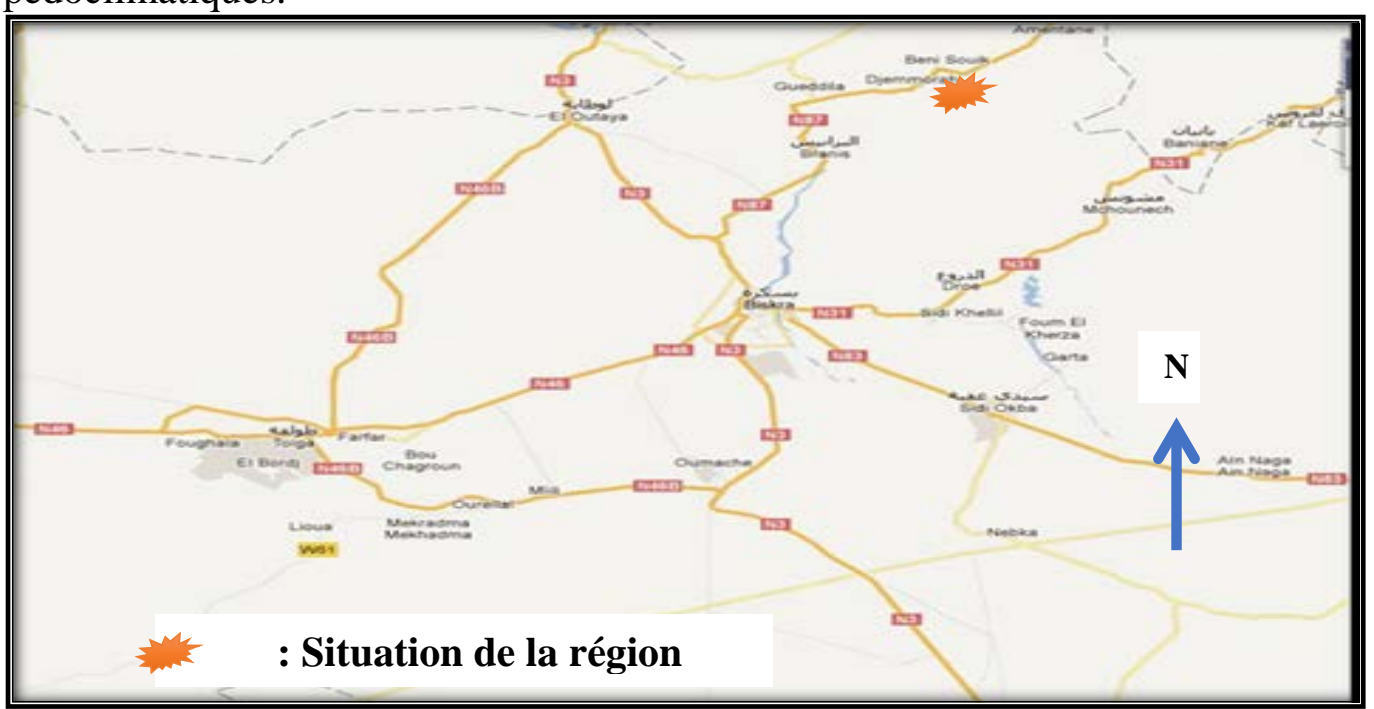

Figure 1 : Situation géographique de la région d'étude.

\section{Méthodes :}

L’objectif de cette étude est de mettre en exergue le développement de l'abricotier dans le sud est algérien. Il s'articule sur les observations et les 
mesures effectuées sur un échantillon de cinquante fleurs pour chaque variété. Les rendements quantitatifs sont évalués sur une dizaine d'arbres pris aléatoirement des vergers étudiés.

Les prélèvements d'échantillons sont accomplis conformément aux consignes indiquées par le descripteur spécifique à l'abricotier (UPOV 2008), élaboré par l'union européenne pour la production et l'obtention végétale (Audergon et al., 1989 et Grimplet J., 2004).

Les résultats obtenus sont analysés par le logiciel STATISTICA 6.

\section{Résultats et discussion :}

\subsection{L'arbre :}

La vigueur de l'arbre de chaque variété est évaluée par la croissance des jeunes pousses, du port de l'arbre et de son degré de ramification. Les résultats d'observations après comparaison à ceux illustrés dans le descripteur de l’UPOV (2008) sont indiqués dans le Tableau 1.

Tableau 1 : Appréciation des arbres des variétés étudiées.

\begin{tabular}{|c|c|c|c|}
\hline Variétés & Vigueur & Port & Degré de ramification \\
\hline Arbi & Moyenne & Etalé & Moyen \\
\hline Boufarik & Moyenne & Etalé & Moyen \\
\hline Badai & Moyenne & Etalé & Moyen \\
\hline Pêche de Nancy & Très Forte & Etalé & Fort \\
\hline
\end{tabular}

\subsection{Les fleurs :}

\subsubsection{Les stades phénologiques :}

Les principaux stades repères de floraison pour cette espèce sont illustrés dans la figure 2.

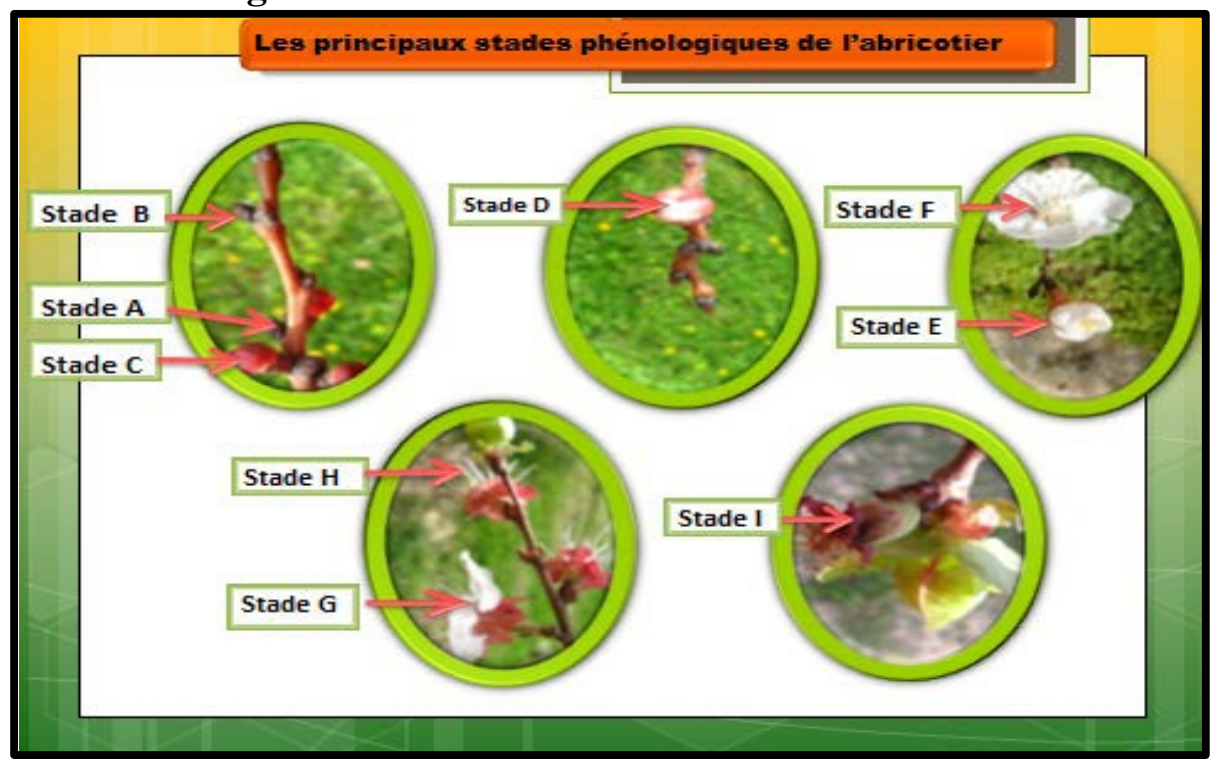

Figure 2 : Les principaux stades repères de floraison chez l’abricotier. 
La floraison de l'abricotier dans cette région précède la foliaison de quelques jours. Les résultats obtenus pour la durée de la floraison sont indiqués dans la Figure 3.

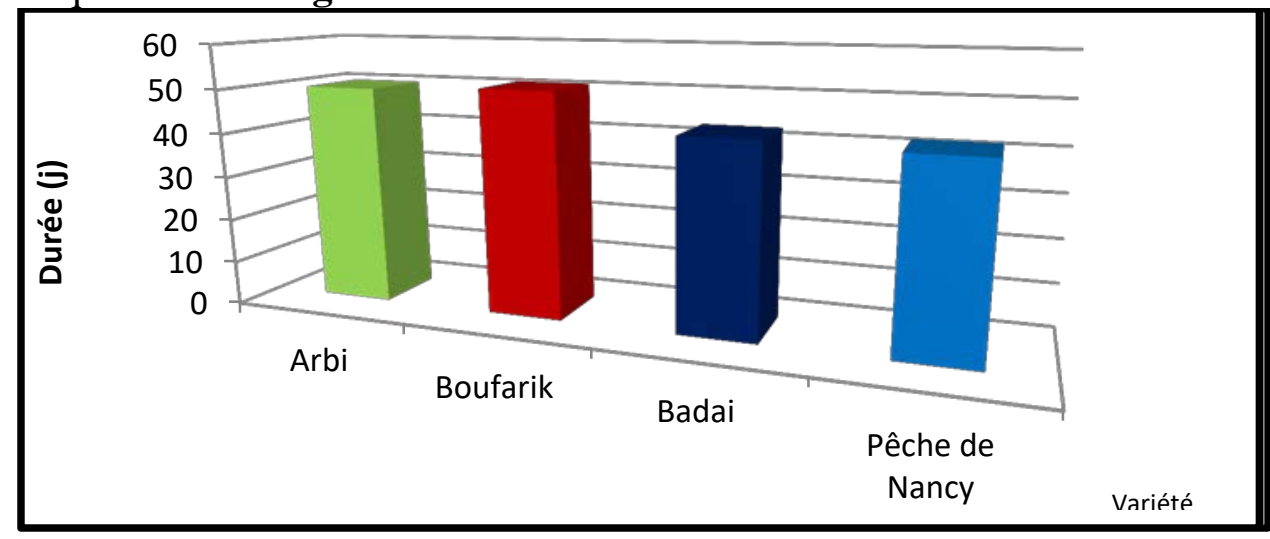

Figure 3 : Variation de la durée moyenne de floraison chez les variétés étudiées.

Concernant les variétés étudiées, la pleine floraison (stade F) s'échelonne de mi-février jusqu'à fin mars (Benaziza A. et Lebid H., 2007). Nos observations vont dans le même sens que ceux indiquées par GAUTIER (1971). Ce paramètre peut varier de 8 à 12 jours selon les exigences variétales et les conditions climatiques de la culture. La durée entre le stade $\mathbf{B}$ et le stade $\mathbf{I}$ varie de 43 à 51 jours quel que soit la variété considérée (Benaziza A., 2011 et Benaziza A. et Makhloula S., 2012).

\subsubsection{Caractères floraux :}

Les paramètres floraux étudiés sont essentiellement le diamètre, la longueur et la largeur des pétales ainsi que la position du carpelle par rapport aux anthères (Benaziza A. et Makhloula S., 2012).

L'aspect général des fleurs des variétés étudiées est indiqué dans la figure 4 et celui des pétales dans la figure 5 .

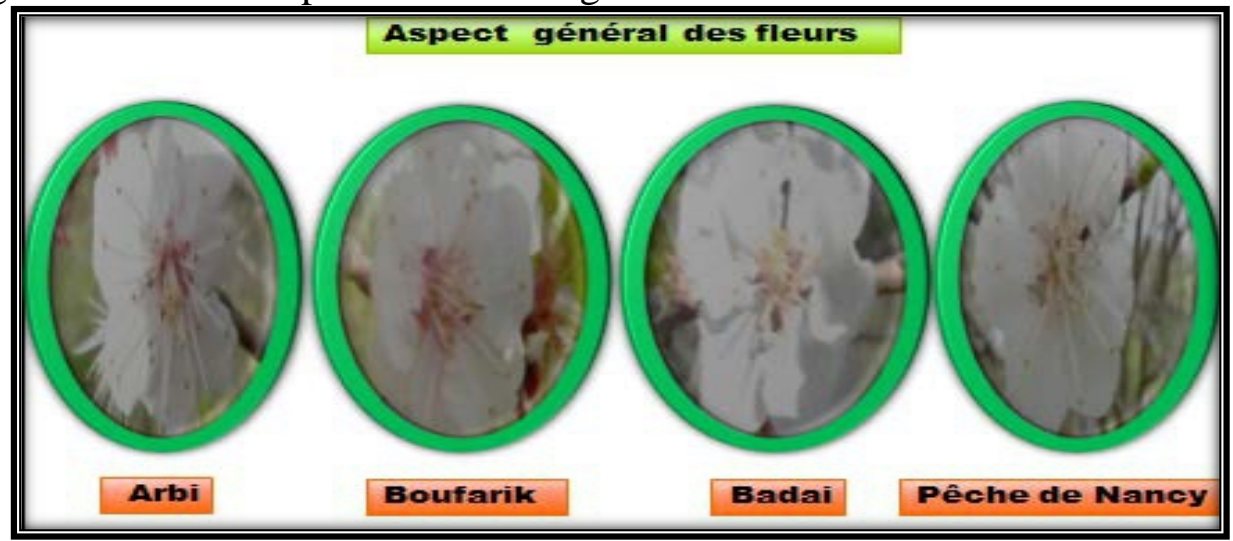

Figure 4 : Aspect général des fleurs des variétés étudiées. 


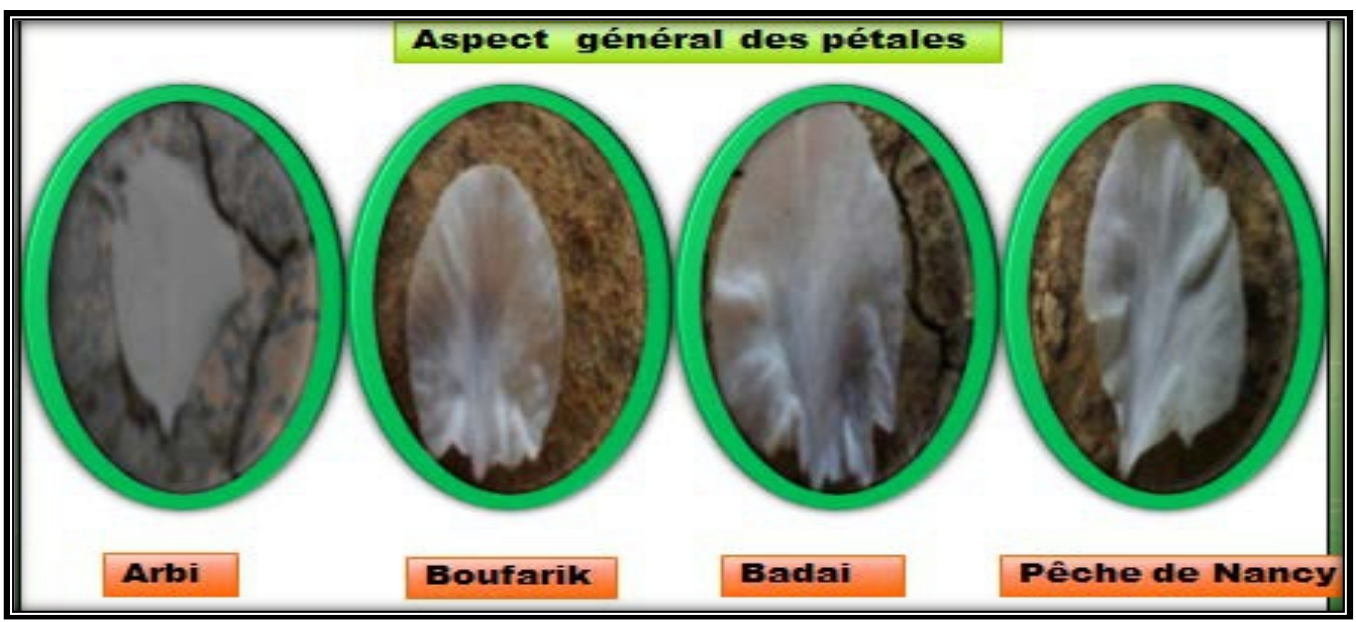

Figure 5 : Aspect général des pétales des fleurs des variétés étudiées.

\subsubsection{Diamètre des fleurs :}

Le diamètre des fleurs est un paramètre déterminant de la pollinisation, il indique l'ampleur de la réceptivité des fleurs par rapport aux agents pollinisateurs Benaziza A., 2011). Les mesures effectuées sont illustrées dans le Tableau 2 et la Figure 6.

Tableau 2 : Analyse descriptive du diamètre moyen des fleurs des variétés étudiées.

\begin{tabular}{|c|c|c|c|c|}
\hline $\begin{array}{c}\text { Variétés / } \\
\text { caractères }\end{array}$ & $\begin{array}{c}\text { Effectif } \\
\mathbf{N}\end{array}$ & $\begin{array}{c}\text { Moyenne } \\
\text { (cm) }\end{array}$ & $\begin{array}{c}\text { Écart } \\
\text { type }\end{array}$ & $\begin{array}{c}\text { Coefficient de } \\
\text { Variation (\%) }\end{array}$ \\
\hline Arbi & 50 & 3,10 & 0,23 & 7,41 \\
\hline Boufarik & 50 & 3,31 & 0,22 & 6,64 \\
\hline Badai & 50 & 3,35 & 0,28 & 8,35 \\
\hline Pêche de Nancy & 50 & 3,65 & 0,30 & 8,21 \\
\hline
\end{tabular}

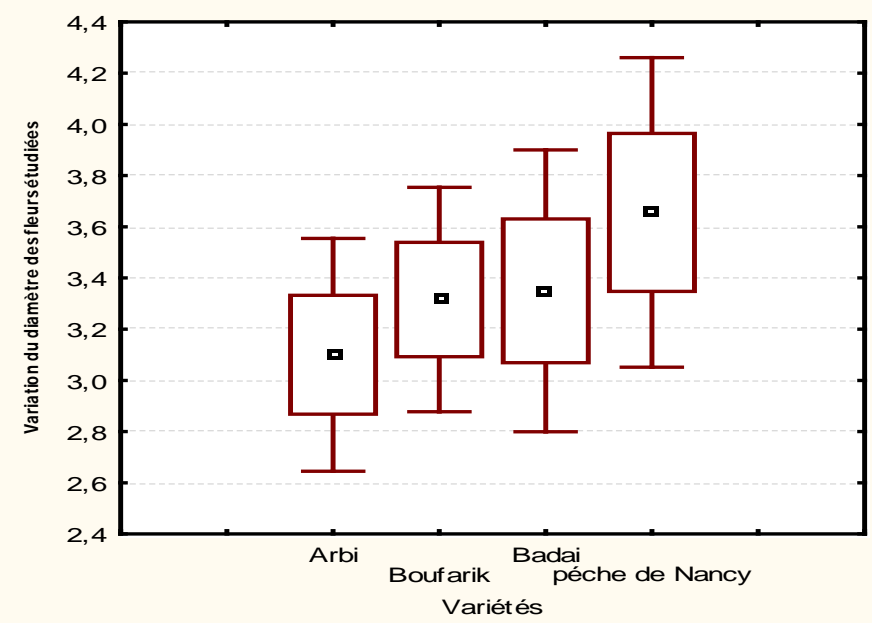

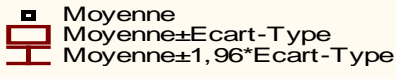

Figure 6 : Variation du diamètre moyen des fleurs des variétés étudiées $(\mathrm{cm})$. 
Ces données exhibent que les fleurs des variétés étudiées détiennent des diamètres différents. La variété Pêche de Nancy possède des fleurs les plus larges par rapport aux autres types de fleurs, ce qui se répercute forcément sur la pollinisation des fleurs et par conséquence sur la production. En revanche la variété Arbi possède les fleurs les plus courtes.

Le coefficient de variation pour ce paramètre est aussi fonction des variétés ; il est plus important chez la variété Badai (variété plus variable pour ce facteur) et faible pour la variété Boufarik (variété la plus stable). Les différences sont significatives du point de vue statistique au seuil $\mathbf{5 \%}$ et corrèle avec la longueur des pétales $(\mathbf{r}=\mathbf{0 . 7 9}, \mathbf{r}=\mathbf{0 . 7 3}, \mathbf{r}=\mathbf{0 . 8 8}$ et $\mathbf{r}=\mathbf{0 , 8 2})$ respectivement chez les variétés Arbi, Boufarik, Badai et Pêche de Nancy et la largeur des pétales $(\mathbf{r}=\mathbf{0 . 5 8}, \mathbf{r}=\mathbf{0 . 8 1})$ chez les variétés Arbi et Pêche de Nancy.

\subsubsection{Longueur des pétales :}

C'est aussi un paramètre de description variétal. Les mesures effectuées sont référées dans le tableau 3 et la figure 7.

Tableau 3 : Analyse descriptive de la longueur moyenne des pétales des variétés étudiées.

\begin{tabular}{|c|c|c|c|c|c|c|c|}
\hline \multicolumn{4}{|c|}{ Variétés / caractères } & $\begin{array}{l}\text { Effect } \\
\text { if } N\end{array}$ & $\begin{array}{l}\text { Moyenne } \\
(\mathrm{cm})\end{array}$ & $\begin{array}{l}\text { Écart } \\
\text { type }\end{array}$ & $\begin{array}{c}\text { Coefficient de } \\
\text { Variation (\%) }\end{array}$ \\
\hline \multicolumn{4}{|c|}{ Arbi } & 50 & 1,31 & 0,10 & 7,6 \\
\hline \multicolumn{4}{|c|}{ Boufarik } & 50 & 1,32 & 0,12 & 9,09 \\
\hline \multicolumn{4}{|c|}{ Badai } & 50 & 1,44 & 0,15 & 10,4 \\
\hline $\begin{array}{l}\text { Pêche de } \\
\text { Nancy }\end{array}$ & 50 & 1,56 & 0,17 & & & 0,8 & \\
\hline
\end{tabular}
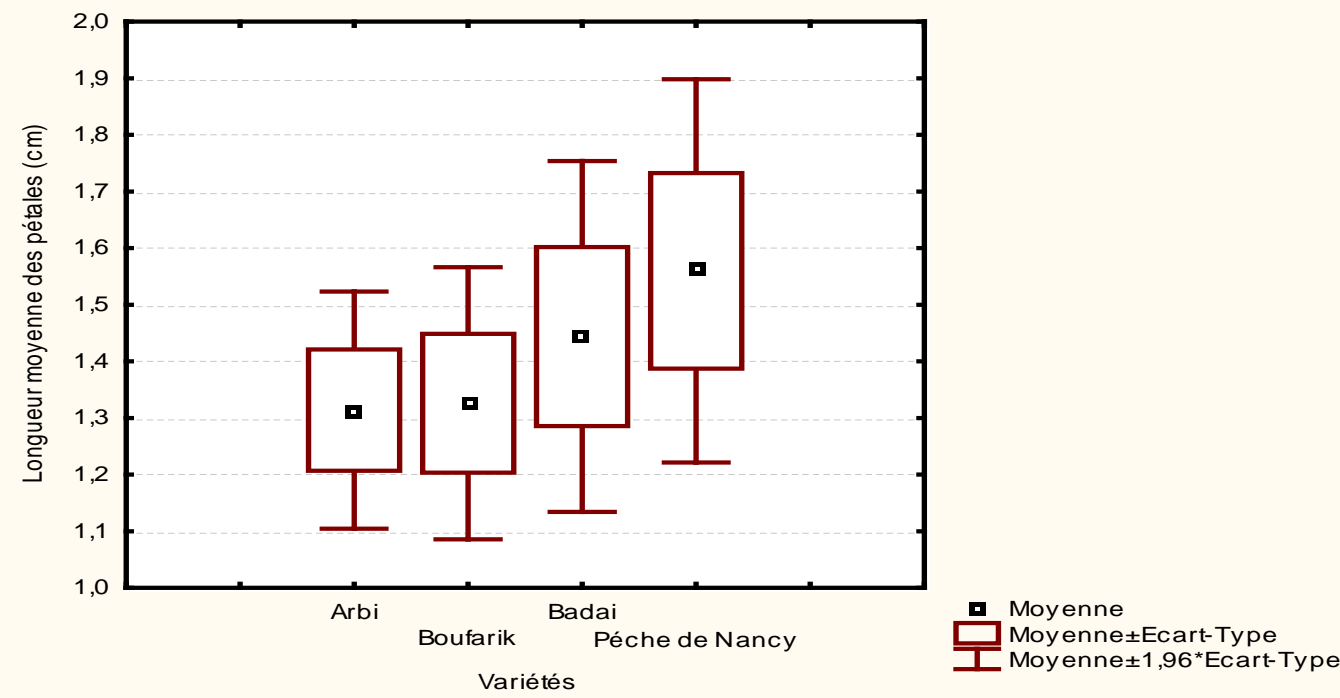

Figure 7 : Longueur moyenne des pétales des variétés étudiées (cm). 


\subsubsection{La largeur des pétales :}

Évidemment comme les facteurs précédents, la largeur des pétales est fonction des variétés. Les résultats obtenus sont récapitulés dans le tableau 4 et la figure 8.

Tableau 4 : Analyse descriptive de la largeur moyenne des pétales des variétés étudiées.

\begin{tabular}{|c|c|c|c|c|}
\hline $\begin{array}{c}\text { Variétés / } \\
\text { caractères }\end{array}$ & $\begin{array}{c}\text { Effectif } \\
\mathbf{N}\end{array}$ & $\begin{array}{c}\text { Moyenne } \\
\text { (cm) }\end{array}$ & $\begin{array}{c}\text { Écart } \\
\text { type }\end{array}$ & $\begin{array}{c}\text { Coefficient de } \\
\text { Variation (\%) }\end{array}$ \\
\hline Arbi & 50 & 1,47 & 0,08 & 5,44 \\
\hline Boufarik & 50 & 1,52 & 0,15 & 9,86 \\
\hline Badai & 50 & 1,76 & 0,12 & 6,81 \\
\hline Pêche de Nancy & 50 & 1,77 & 0,15 & 8,47 \\
\hline
\end{tabular}

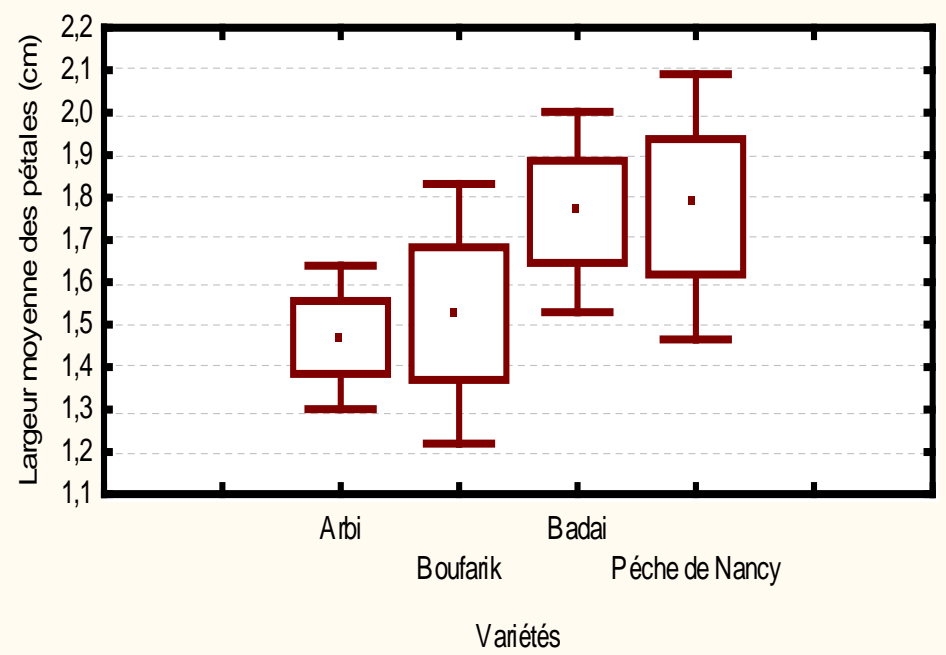

Figure 8 : Largeur moyenne des pétales des variétés étudiées (cm).

Les variétés pêche de Nancy et Badai se démarquent par des pétales les plus larges ce qui favorise en plus la pollinisation des fleurs. Par contre ceux de la variété Arbi sont les plus courts. Le coefficient de variation est plus élevé chez les variétés Boufarik et pêche de Nancy.

L'analyse statistique montre des différences significatives du point de vue statistique au seuil $\mathbf{5 \%}$. Ce Paramètre est corrélé positivement avec le diamètre de la fleur $(\mathbf{r}=\mathbf{0 . 5 8}, \mathbf{r}=\mathbf{0 . 7 4}$ et $\mathbf{r}=\mathbf{0 . 8 1})$ respectivement chez les variétés Arbi, Boufarik et Pêche de Nancy. Il est à signaler que chez la variété Badai les différences ne sont pas significatives.

\subsubsection{Position du carpelle par rapport aux étamines :}

La position du carpelle par rapport aux anthères des fleurs des variétés étudiées est illustrée dans la figure 9. 

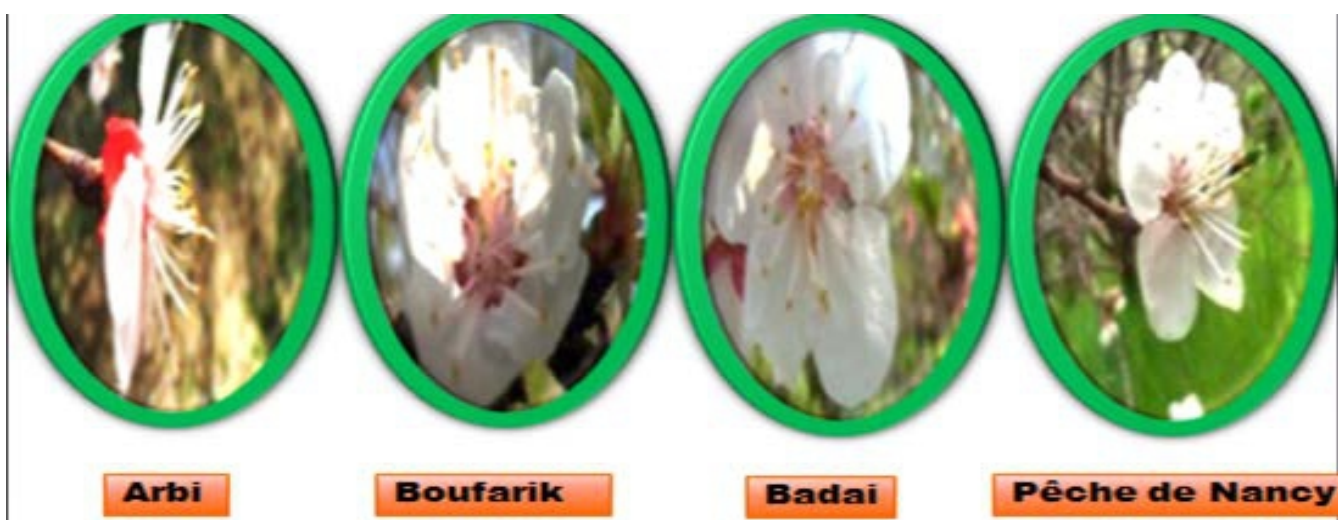

Figure 9 : Position des carpelles par rapport aux anthères chez les fleurs des variétés étudiées.

La position du carpelle par rapport aux étamines est étudiée pour la première fois pour cette espèce. Cette caractéristique revêt une importance particulière quant à la pollinisation et à la production. Un carpelle situé audessus des anthères nécessite obligatoirement des agents pollinisateurs assurant le transport des grains de pollens pour assurer la fécondation des ovules ; cas des fleurs des quatre variétés étudiées qui exigent forcément des agents pollinisateurs, c'est pourquoi ces variétés sont considérées comme inter fertiles.

\section{Rendements :}

Les rendements sont appréciés à partir de la production moyenne des arbres et de la densité de plantation réelle de chaque variété. Les résultats obtenus sont récapitulés dans la figure 10.

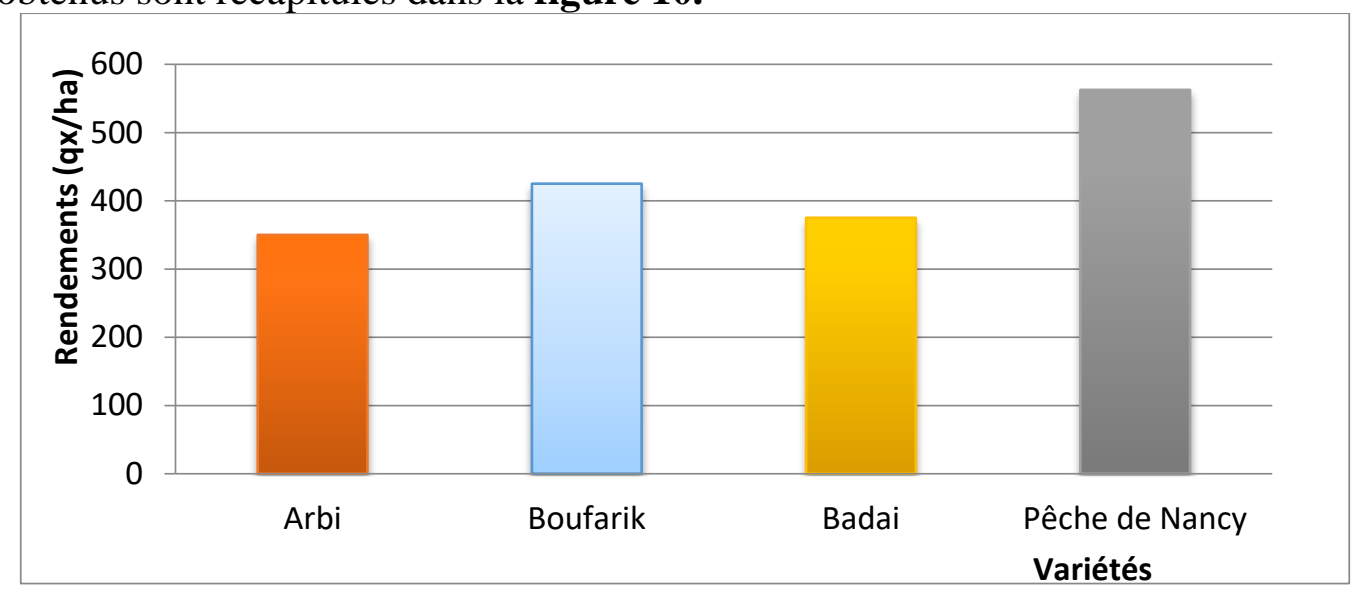

Figure 10 : Les rendements calculés chez les variétés étudiées.

Ces résultats met en exergue la production moyenne par hectare qui est globalement encourageante particulièrement chez les variétés Pêche de 
Nancy et Boufarik qui marquent des rendements respectivement de 562.5 qx / ha et 425 qx/ha. Ces dernières variétés sont d'ailleurs les mieux sélectionnées par les agriculteurs pour un meilleur développement de cette espèce dans la région. Ces aboutissements demeurent préliminaires et prouvent l’importance de la culture dans cette zone.

\section{Conclusion :}

Au terme de ce travail axé sur la caractérisation florale et l'évaluation des rendements de quatre variétés d'abricotier, nous avons pu parvenir aux conclusions suivantes :

$\checkmark$ L’appréciation de la vigueur évaluée à partir de la longueur des jeunes pousses indique que les variétés Badai et Pêche de Nancy sont plus vigoureuses par rapport aux deux autres variétés.

$\checkmark$ Concernant la phénologie nous avons pu mettre en évidence la précocité de floraison de ces variétés qui sont en relation directe avec les conditions climatiques de la région. Ce qui présente un intérêt agro économique de la culture.

$\checkmark$ L’analyse des différents paramètres floraux montre que les fleurs sont plus larges chez la variété Pêche de Nancy. Ce qui nécessite forcément des agents de pollinisation.

$\checkmark$ Les observations relatives à la position du carpelle par rapport aux anthères sont à soulever. Cette particularité est la première fois indiquée chez cette espèce. Elle est aussi en relation étroite avec le phénomène de la pollinisation et par conséquent sur les rendements, ce qui requiert un moyen de pollinisation comme par exemple l'installation des ruches d'abeille.

$\checkmark$ Du point de vue productivité, ces variétés s’avèrent plus productives et présentent alors des caractéristiques distinctives d'une variété à une autre. Les variétés pêche de Nancy et Boufarik se montrent plus productives par rapport aux deux autres.

$\checkmark$ Malgré les résultats obtenus, ils demeurent préliminaires et constituent le point focal d'études antérieures dans la région et met en évidence les différentes interactions entre cette espèce et les conditions pédoclimatiques locales. Par ailleurs, cette étude montre clairement les performances de l'abricotier indiquées à travers les caractères floraux et les rendements de chaque variété, ce qui requiert un intérêt particulier à la culture pour un meilleur développement socio-économique local et régional.

\section{References:}

1. AUDERGON J.M., SOUTY M., BREUILS L., REICH M. et DUFFILOL J.M., 1989. Recherches sur les déterminismes génétiques associés aux paramètres de qualité des fruits chez l’abricot. INRA, Avignon, pp. 89-97. 
2. BENAZIZA A. and H. LEBID , 2007. Caractérisation de quelques varieties d'abricotier (Prunus armenaica L.) dans la region de M'chounèche- mem. Biskra, Algérie 107p.

3. BENAZIZA A., 2011. Behavior and morphological, physiological and agronomics studies of some varieties of apricot grown in the oasis environment 87p.

4. BENAZIZA A. et MAKHLOULA S., 2012. Comportement de quelques variétés d'abricotier cultivées dans la région de Djamorah. Mém. Master biologie 92p.

5. BENOUR L., 2004. Amélioration variétale de l'abricotier. Edit. INRA France, $43 \mathrm{p}$.

6. GRIMPLET J., 2004. Génomique fonctionnelle et marqueurs de qualité chez l'abricot. Thèse Doct. IN.P Toul, 253 p.

7. UPOV, 2005. Principes directeurs pour la conduite de l'examen de la distinction, de l'homogénéité et de la stabilité Union européenne pour la protection et l'obtention du matériel végétal. UPOV, Edit. INRA France, 41p. 